TRANSACTIONS OF THE

AMERICAN MATHEMATICAL SOCIETY

Volume 360, Number 6, June 2008, Pages 3107-3119

S 0002-9947(08)04427-9

Article electronically published on January 8, 2008

\title{
A CONNECTEDNESS RESULT IN POSITIVE CHARACTERISTIC
}

\author{
ANURAG K. SINGH AND ULI WALTHER
}

Dedicated to Professor Paul Roberts on the occasion of his sixtieth birthday

\begin{abstract}
Let $(R, \mathfrak{m})$ be a complete local ring of dimension at least two, which contains a separably closed coefficient field of positive characteristic. Using a vanishing theorem of Peskine-Szpiro, Lyubeznik proved that the local cohomology module $H_{\mathfrak{m}}^{1}(R)$ is Frobenius-torsion if and only if the punctured spectrum of $R$ is connected in the Zariski topology. We give a simple proof of this theorem and, more generally, a formula for the number of connected components in terms of the Frobenius action on $H_{\mathfrak{m}}^{1}(R)$.
\end{abstract}

\section{INTRODUCTION}

All rings considered in this note are commutative and Noetherian. We give a simple proof of the following result due to Lyubeznik:

Theorem 1.1 ([Ly2, Corollary 4.6]). Let $(R, \mathfrak{m})$ be a complete local ring of dimension at least two, with a separably closed coefficient field of positive characteristic. Then the e-th iteration of the Frobenius map

$$
F: H_{\mathfrak{m}}^{1}(R) \rightarrow H_{\mathfrak{m}}^{1}(R)
$$

is zero for $e \gg 0$ if and only if $\operatorname{Spec} R \backslash\{\mathfrak{m}\}$ is connected in the Zariski topology.

We also obtain, by similar methods, the following theorem:

Theorem 1.2. Let $(R, \mathfrak{m})$ be a complete local ring of positive dimension, with an algebraically closed coefficient field of positive characteristic. Then the number of connected components of $\operatorname{Spec} R \backslash\{\mathfrak{m}\}$ is

$$
1+\operatorname{dim}_{K} \bigcap_{e \in \mathbb{N}} F^{e}\left(H_{\mathfrak{m}}^{1}(R)\right) .
$$

In Section 5 we describe how this provides an algorithm to determine the number of geometrically connected components of projective algebraic sets defined over a finite field: computer algebra algorithms for primary decomposition can be used to determine the number of connected components over finite extensions of the fields $\mathbb{F}_{p}$ or $\mathbb{Q}$, but not over the algebraic closures of these fields. In the case of characteristic zero, de Rham cohomology allows for the computation of the number

Received by the editors March 26, 2006.

2000 Mathematics Subject Classification. Primary 13D45; Secondary 13A35.

The first author was supported by NSF grants DMS 0300600 and DMS 0600819.

The second author was supported by NSF grants DMS 0100509 and DMS 0555319, and by NSA grant H98230-06-1-0012. We are grateful to Gennady Lyubeznik for useful discussions and comments. 
of geometrically connected components via $D$-module methods, [Wal], and we show that the Frobenius provides analogous methods in the case of positive characteristic.

Theorem 1.1 is obtained in [Ly2] as a corollary of the following two theorems of Lyubeznik and Peskine-Szpiro:

Theorem 1.3 ([Ly2, Theorem 1.1]). Let $(A, \mathfrak{M})$ be a regular local ring containing a field of positive characteristic, and let $\mathfrak{A}$ be an ideal of $A$. Then $H_{\mathfrak{A}}^{i}(A)=0$ if and only if there exists an integer $e \geq 1$ such that the $e$-th Frobenius iteration

$$
F^{e}: H_{\mathfrak{M}}^{\operatorname{dim}}{ }^{-i}(A / \mathfrak{A}) \rightarrow H_{\mathfrak{M}}^{\operatorname{dim}}{ }^{A-i}(A / \mathfrak{A})
$$

is the zero map.

Theorem 1.4 ([PS, Chapter III, Theorem 5.5]). Let $(A, \mathfrak{M})$ be a complete regular local ring with a separably closed coefficient field of positive characteristic, and let $\mathfrak{A}$ be an ideal of $A$. Then $H_{\mathfrak{A}}^{i}(A)=0$ for $i \geq \operatorname{dim} A-1$ if and only if $\operatorname{dim}(A / \mathfrak{A}) \geq 2$ and $\operatorname{Spec}(A / \mathfrak{A}) \backslash\{\mathfrak{M}\}$ is connected.

Our proof of Theorem 1.1 is "simple" in the sense that it does not rely on vanishing theorems such as those of $[\mathrm{PS}]$ —indeed, the only ingredient, aside from elementary considerations, is the local duality theorem. Results analogous to Theorem 1.4 were proved by Hartshorne in the projective case [HaR, Theorem 7.5], and by Ogus in equicharacteristic zero using de Rham cohomology [Og, Corollary 2.11]. Combining these results, one has:

Theorem 1.5. Let $(A, \mathfrak{M})$ be a regular local ring containing a field, and let $\mathfrak{A}$ be an ideal of $A$. Then $H_{\mathfrak{A}}^{i}(A)=0$ for $i \geq \operatorname{dim} A-1$ if and only if

(1) $\operatorname{dim}(A / \mathfrak{A}) \geq 2$, and

(2) $\operatorname{Spec}(A / \mathfrak{A}) \backslash\{\mathfrak{M}\}$ is formally geometrically connected (see Definition 2.1).

Huneke and Lyubeznik [HL, Theorem 2.9] gave a characteristic free proof of this using a generalization of a result of Faltings, [Fa, Satz 1]. Some other applications of local cohomology theory which yield strong results on the connectedness properties of algebraic varieties may be found in the papers $[\mathrm{BR}]$ and $[\mathrm{HH}]$, where the authors obtain generalizations of Faltings' connectedness theorem.

For the convenience of the reader, we include an Appendix with some facts about Frobenius actions; see Section 6.

\section{PRELiminary REMARKS}

Notation. When $R$ is the homomorphic image of a ring $A$, we use upper-case letters $\mathfrak{P}, \mathfrak{Q}, \mathfrak{M}, \mathfrak{A}, \mathfrak{B}$ for ideals of $A$, and corresponding lower-case letters $\mathfrak{p}, \mathfrak{q}, \mathfrak{m}, \mathfrak{a}, \mathfrak{b}$ for their images in $R$.

Definition 2.1. Let $(R, \mathfrak{m})$ be a local ring. A field $K \subseteq R$ is a coefficient field for $R$ if the composition $K \hookrightarrow R \rightarrow R / \mathfrak{m}$ is an isomorphism. Every complete local ring containing a field has a coefficient field.

We recall some notions from [Ra, Chapitre VIII]. Let $(R, \mathfrak{m}, K)$ be a local ring and let $\overline{f(T)} \in K[T]$ denote the image of a polynomial $f(T) \in R[T]$. Then $R$ is Henselian if for every monic polynomial $f(T) \in R[T]$, every factorization of $\overline{f(T)}$ as a product of relatively prime monic polynomials in $K[T]$ lifts to a factorization of $f(T)$ as a product of monic polynomials in $R[T]$. Hensel's Lemma is precisely the statement that every complete local ring is Henselian. The Henselization of a local 
ring $R$ is a local ring $R^{\mathrm{h}}$, with the property that every local homomorphism from $R$ to a Henselian local ring factors uniquely through $R^{\mathrm{h}}$. The ring $R^{\mathrm{h}}$ is obtained by taking the direct limit of all local étale extensions $S$ of $R$ for which $(R, \mathfrak{m}) \rightarrow(S, \mathfrak{n})$ induces an isomorphism of residue fields $R / \mathfrak{m} \cong S / \mathfrak{n}$.

A local ring $(R, \mathfrak{m}, K)$ is said to be strictly Henselian if it is Henselian and its residue field $K$ is separably closed. It is easily seen that $R$ is strictly Henselian if and only if every monic polynomial $f(T) \in R[T]$ for which $\overline{f(T)} \in K[T]$ is separable splits into linear factors in $R[T]$. Every local ring has a strict Henselization $R^{\text {sh }}$, such that every local homomorphism from $R$ to a strictly Henselian ring factors through $R^{\mathrm{sh}}$. The strict Henselization of a field $K$ is its separable closure $K^{\text {sep }}$. In general, the strict Henselization of a local ring $(R, \mathfrak{m}, K)$ is obtained by fixing an embedding $\iota: K \rightarrow K^{\text {sep }}$, and taking the direct limit of local étale extensions $(S, \mathfrak{n}, L)$ of $(R, \mathfrak{m}, K)$ with $L \hookrightarrow K^{\text {sep }}$, for which the induced map $K \rightarrow L \rightarrow K^{\text {sep }}$ agrees with $\iota: K \rightarrow K^{\text {sep }}$.

The punctured spectrum of a local ring $(R, \mathfrak{m})$ is the set Spec $R \backslash\{\mathfrak{m}\}$, with the topology induced by the Zariski topology on $\operatorname{Spec} R$. We say that the punctured spectrum of $R$ is formally geometrically connected if the punctured spectrum of $\hat{R}^{\text {sh }}$, the completion of the strict Henselization of the completion of $R$, is connected. If $R$ is an $\mathbb{N}$-graded ring which is finitely generated over a field $R_{0}=K$, then Proj $R$ is said to be geometrically connected if $\operatorname{Proj}\left(R \otimes_{K} K^{\mathrm{sep}}\right)$ is connected.

Definition 2.2. Let $\mathfrak{a}$ be an ideal of a ring $R$. A ring homomorphism $\varphi: R \rightarrow S$ induces a map of local cohomology modules $H_{\mathfrak{a}}^{i}(R) \stackrel{\varphi}{\rightarrow} H_{\mathfrak{a} S}^{i}(S)$. In particular, if $R$ contains a field of characteristic $p>0$, then the Frobenius homomorphism $F: R \rightarrow R$ induces an additive map

$$
H_{\mathfrak{a}}^{i}(R) \stackrel{F}{\rightarrow} H_{\mathfrak{a}[p]}^{i}(R)=H_{\mathfrak{a}}^{i}(R),
$$

called the Frobenius action on $H_{\mathfrak{a}}^{i}(R)$. An element $\eta \in H_{\mathfrak{a}}^{i}(R)$ is F-torsion if $F^{e}(\eta)=0$ for some $e \in \mathbb{N}$. The module $H_{\mathfrak{a}}^{i}(R)$ is $F$-torsion if each element is $F$-torsion. The image of $F^{e}$ need not be an $R$-module, but it is a $K$-vector space when $K$ is perfect. In this case the $F$-stable part of $H_{\mathfrak{a}}^{i}(R)$ is the vector space

$$
H_{\mathfrak{a}}^{i}(R)_{\mathrm{st}}=\bigcap_{e \in \mathbb{N}} F^{e}\left(H_{\mathfrak{a}}^{i}(R)\right) .
$$

Some results about $F$-torsion modules and $F$-stable subspaces are summarized in Section 6. For a very general theory of $F$-modules, we refer the reader to [Ly1].

Remark 2.3. Consider a local ring $(R, \mathfrak{m})$ of positive dimension. The punctured spectrum of $R$ is disconnected if and only if the minimal primes of $R$ can be partitioned into two sets $\mathfrak{p}_{1}, \ldots, \mathfrak{p}_{m}$ and $\mathfrak{q}_{1}, \ldots, \mathfrak{q}_{n}$ such that $\operatorname{rad}\left(\mathfrak{p}_{i}+\mathfrak{q}_{j}\right)=\mathfrak{m}$ for all pairs $\mathfrak{p}_{i}, \mathfrak{q}_{j}$. Consider the graph $\Gamma$ whose vertices are the minimal primes of $R$, and there is an edge between minimal primes $\mathfrak{p}$ and $\mathfrak{p}^{\prime}$ if and only if $\operatorname{rad}\left(\mathfrak{p}+\mathfrak{p}^{\prime}\right) \neq \mathfrak{m}$. It follows that the punctured spectrum of $R$ is connected if and only if the graph $\Gamma$ is connected. If the graph $\Gamma$ is connected, take a spanning tree, i.e., a connected acyclic subgraph, containing all the vertices of $\Gamma$. This spanning tree must contain a vertex $\mathfrak{p}_{i}$ with only one edge, so $\Gamma \backslash\left\{\mathfrak{p}_{i}\right\}$ is connected as well.

Let $\mathfrak{P}_{1}, \ldots, \mathfrak{P}_{n}$ be incomparable prime ideals of a local domain $A$. Then their images $\mathfrak{p}_{1}, \ldots, \mathfrak{p}_{n}$ are precisely the minimal primes of the ring $R=A /\left(\mathfrak{P}_{1} \cap \cdots \cap \mathfrak{P}_{n}\right)$. From the above discussion, we conclude that if the punctured spectrum of $R$ is 
connected, then there exists $i$ such that the punctured spectrum of the ring

$$
A /\left(\mathfrak{P}_{1} \cap \cdots \cap \hat{\mathfrak{P}}_{i} \cap \cdots \cap \mathfrak{P}_{n}\right)
$$

is connected as well.

Theorems 1.1 and 1.2 assert that connectedness issues for $\operatorname{Spec} R \backslash\{\mathfrak{m}\}$ are determined by the Frobenius action on $H_{\mathfrak{m}}^{1}(R)$. We next record an observation about the length of $H_{\mathfrak{m}}^{1}(R)$.

Proposition 2.4. Let $(R, \mathfrak{m})$ be a local ring which is a homomorphic image of a Gorenstein domain. Then $H_{\mathfrak{m}}^{1}(R)$ has finite length if and only if $\operatorname{ann}_{R} \mathfrak{p}=0$ for every prime ideal $\mathfrak{p}$ of $R$ with $\operatorname{dim} R / \mathfrak{p}=1$.

Proof. If $\operatorname{dim} R=0$, then $H_{\mathfrak{m}}^{1}(R)=0$, and $R$ has no primes with $\operatorname{dim} R / \mathfrak{p}=1$. If $\operatorname{dim} R=1$, then $H_{\mathfrak{m}}^{1}(R)$ has infinite length and $\operatorname{dim} R / \mathfrak{p}=1$ for some minimal prime $\mathfrak{p}$ of $R$. For the rest of the proof we hence assume that $\operatorname{dim} R \geq 2$.

Let $R=A / \mathfrak{Q}$ where $A$ is a Gorenstein domain. Localizing $A$ at the inverse image of $\mathfrak{m}$, we may assume that $(A, \mathfrak{M})$ is a local ring. Using local duality over $A$, the module $H_{\mathfrak{m}}^{1}(R)=H_{\mathfrak{M}}^{1}(A / \mathfrak{Q})$ has finite length if and only if $\operatorname{Ext}_{A}^{\operatorname{dim}} A-1(A / \mathfrak{Q}, A)$ has finite length as an $A$-module. Since $\operatorname{Ext}_{A}^{\operatorname{dim} A-1}(A / \mathfrak{Q}, A)$ is finitely generated, this is equivalent to the vanishing of

$$
\operatorname{Ext}_{A}^{\operatorname{dim} A-1}(A / \mathfrak{Q}, A)_{\mathfrak{P}}=\operatorname{Ext}_{A_{\mathfrak{P}}}^{\operatorname{dim} A-1}\left(A_{\mathfrak{P}} / \mathfrak{Q} A_{\mathfrak{P}}, A_{\mathfrak{P}}\right)
$$

for all $\mathfrak{P} \in \operatorname{Spec} A \backslash\{\mathfrak{M}\}$. Using local duality over the Gorenstein local ring $\left(A_{\mathfrak{P}}, \mathfrak{P} A_{\mathfrak{P}}\right)$, this is equivalent to the vanishing of

$$
H_{\mathfrak{P} A_{\mathfrak{P}}}^{\operatorname{dim} A_{\mathfrak{P}}-\operatorname{dim} A+1}\left(A_{\mathfrak{P}} / \mathfrak{Q} A_{\mathfrak{P}}\right)=H_{\mathfrak{p} R_{\mathfrak{p}}}^{\operatorname{dim} A_{\mathfrak{P}}-\operatorname{dim} A+1}\left(R_{\mathfrak{p}}\right)
$$

for all $\mathfrak{P} \in \operatorname{Spec} A \backslash\{\mathfrak{M}\}$. This local cohomology module vanishes for $\mathfrak{P} \notin V(\mathfrak{Q})$. Since $\operatorname{dim} A_{\mathfrak{P}}-\operatorname{dim} A+1 \leq 0$ for $\mathfrak{P} \in \operatorname{Spec} A \backslash\{\mathfrak{M}\}$, we need only consider primes $\mathfrak{P} \in V(\mathfrak{Q})$ with $\operatorname{dim} A_{\mathfrak{P}}=\operatorname{dim} A-1$. Since $A$ is a catenary local domain, $\operatorname{dim} A_{\mathfrak{P}}$ equals $\operatorname{dim} A-1$ precisely when $\operatorname{dim} A / \mathfrak{P}=1$, equivalently $\operatorname{dim} R / \mathfrak{p}=1$. Hence $H_{\mathfrak{m}}^{1}(R)$ has finite length if and only if $H_{\mathfrak{p} R_{\mathfrak{p}}}^{0}\left(R_{\mathfrak{p}}\right)=H_{\mathfrak{p}}^{0}(R)$ vanishes for all $\mathfrak{p} \in \operatorname{Spec} R$ with $\operatorname{dim} R / \mathfrak{p}=1$, i.e., if and only if $\operatorname{ann}_{R} \mathfrak{p}=0$ for all $\mathfrak{p}$ with $\operatorname{dim} R / \mathfrak{p}=1$.

\section{MAin RESUlts}

Theorem 3.1. Let $(R, \mathfrak{m})$ be a strictly Henselian local domain containing a field of positive characteristic. If $R$ is a homomorphic image of a Gorenstein domain and $\operatorname{dim} R \geq 2$, then $H_{\mathfrak{m}}^{1}(R)$ is F-torsion.

Proof. Suppose there exists $\eta \in H_{\mathfrak{m}}^{1}(R)$ which is not $F$-torsion. Since $R$ is a domain, Proposition 2.4 implies that $H_{\mathfrak{m}}^{1}(R)$ has finite length. Hence for all integers $e \gg 0$, the element $F^{e}(\eta)$ belongs to the $R$-module spanned by $\eta, F(\eta), F^{2}(\eta), \ldots, F^{e-1}(\eta)$. Amongst all equations of the form

$$
F^{e+k}(\eta)+r_{1} F^{e+k-1}(\eta)+\cdots+r_{e} F^{k}(\eta)=0
$$

with $r_{i} \in R$ for all $i$, choose one where the number of nonzero coefficients $r_{i}$ that occur is minimal. We claim that $r_{e}$ must be a unit. Note that $H_{\mathfrak{m}}^{1}(R)$ is killed by $\mathfrak{m}^{q^{\prime}}$ for some $q^{\prime}=p^{e^{\prime}}$. If $r_{e} \in \mathfrak{m}$, then applying $F^{e^{\prime}}$ to equation (3.1.1), we get

$$
F^{e^{\prime}+e+k}(\eta)+r_{1}^{q^{\prime}} F^{e^{\prime}+e+k-1}(\eta)+\cdots+r_{e}^{q^{\prime}} F^{e^{\prime}+k}(\eta)=0 .
$$


But $r_{e}^{q^{\prime}} F^{e^{\prime}+k}(\eta) \in \mathfrak{m}^{q^{\prime}} H_{\mathfrak{m}}^{1}(R)=0$, so this is an equation with fewer nonzero coefficients, contradicting the minimality assumption. This shows that $r_{e} \in R$ is a unit. Since $\eta$ is not $F$-torsion, neither is $F^{k}(\eta)$, so after replacing $\eta$ if necessary, we have an equation of the form

$$
F^{e}(\eta)+r_{1} F^{e-1}(\eta)+\cdots+r_{e} \eta=0
$$

where $r_{e}$ is a unit and $\eta \in H_{\mathfrak{m}}^{1}(R)$ is not $F$-torsion. Let $\eta=\left[\left(y_{1} / x_{1}, \ldots, y_{d} / x_{d}\right)\right]$ where $H_{\mathfrak{m}}^{1}(R)$ is regarded as the cohomology of a Cech complex on a system of parameters $x_{1}, \ldots, x_{d}$ for $R$. Then (3.1.2) implies that there exists $r_{e+1} \in R$ such that each $y_{i} / x_{i} \in R_{x_{i}}$ is a root of the polynomial

$$
f(T)=T^{p^{e}}+r_{1} T^{p^{e-1}}+\cdots+r_{e} T+r_{e+1} \in R[T] .
$$

Now $f^{\prime}(T)=r_{e}$ is a unit, so $\overline{f(T)} \in R / \mathfrak{m}[T]$ is a separable polynomial. Since $R$ is strictly Henselian, the polynomial $f(T)$ splits in $R[T]$, and hence any root of $f(T)$ in the fraction field of $R$ is an element of $R$. In particular, $y_{1} / x_{1}=\cdots=y_{d} / x_{d}$ is an element of $R$, and so $\eta=0$.

We next prove the connectedness criterion, Theorem 1.1. By Proposition 6.1, the module $H_{\mathfrak{m}}^{1}(R)$ is $F$-torsion if and only if there exists $e$ such that $F^{e}\left(H_{\mathfrak{m}}^{1}(R)\right)=0$. In view of this, the following theorem is equivalent to Theorem 1.1.

Theorem 3.2. Let $(R, \mathfrak{m})$ be a local ring with $\operatorname{dim} R>0$, which contains a field of positive characteristic. Then $H_{\mathfrak{m}}^{1}(R)$ is F-torsion if and only if $\operatorname{dim} R \geq 2$ and the punctured spectrum of $R$ is formally geometrically connected.

Proof. Quite generally, for a local ring $(R, \mathfrak{m})$ we have $H_{\mathfrak{m}}^{i}(\hat{R})=H_{\mathfrak{m}}^{i}(R)$. Moreover, $S=\hat{\hat{R}}^{\text {sh }}$ is a faithfully flat extension of $R$, and $H_{\mathfrak{m}}^{i}(R) \otimes_{R} S \cong H_{\mathfrak{m} S}^{i}(S)$ is $F$-torsion if and only if $H_{\mathfrak{m}}^{i}(R)$ is $F$-torsion. Hence we may assume that $R$ is a complete local ring with a separably closed coefficient field.

Suppose that $H_{\mathfrak{m}}^{1}(R)$ is $F$-torsion. The local cohomology module $H_{\mathfrak{m}}^{\operatorname{dim} R}(R)$ is not $F$-torsion by Proposition 6.2 , so $\operatorname{dim} R \geq 2$. Let $\mathfrak{a}$ and $\mathfrak{b}$ be ideals of $R$ such that $\mathfrak{a}+\mathfrak{b}$ is $\mathfrak{m}$-primary and $\mathfrak{a} \cap \mathfrak{b}=0$. Let

$$
x_{1}=y_{1}+z_{1}, \quad \ldots, \quad x_{d}=y_{d}+z_{d}
$$

be a system of parameters for $R$ where $y_{i} \in \mathfrak{a}$ and $z_{i} \in \mathfrak{b}$. Since $\mathfrak{a} \mathfrak{b} \subseteq \mathfrak{a} \cap \mathfrak{b}=0$, we have $y_{i} z_{j}=0$ for all $i, j$, and hence

$$
y_{i}\left(y_{j}+z_{j}\right)=y_{j}\left(y_{i}+z_{i}\right) .
$$

These relations give an element of $H_{\mathfrak{m}}^{1}(R)$ regarded as the cohomology of a Čech complex on $x_{1}, \ldots, x_{d}$, namely

$$
\eta=\left[\left(\frac{y_{1}}{x_{1}}, \ldots, \frac{y_{d}}{x_{d}}\right)\right] \in H_{\mathfrak{m}}^{1}(R) .
$$

The hypotheses imply that $F^{e}(\eta)=0$ for some $e$, so there exists $q=p^{e}$ and $r \in R$ such that $\left(y_{i} / x_{i}\right)^{q}=r$ in $R_{x_{i}}$ for all $1 \leq i \leq d$. Hence there exists $t \in \mathbb{N}$ such that $x_{i}^{t} y_{i}^{q}=r x_{i}^{q+t}$, i.e.,

$$
\left(y_{i}+z_{i}\right)^{t} y_{i}^{q}=r\left(y_{i}+z_{i}\right)^{q+t} .
$$

But $y_{i} z_{i}=0$, so these equations simplify to give $(1-r) y_{i}^{q+t}=r z_{i}^{q+t}$. Since $R$ is a local ring, either $r$ or $1-r$ must be a unit. If $r$ is a unit, then $z_{i}^{q+t} \in \mathfrak{a}$ for all $i$, 
and so $\mathfrak{a}$ is $\mathfrak{m}$-primary. Similarly if $1-r$ is a unit, then $\mathfrak{b}$ is $\mathfrak{m}$-primary. This proves that the punctured spectrum of $R$ is connected.

For the converse, assume that $\operatorname{dim} R \geq 2$ and that the punctured spectrum of $R$ is connected. Let $\mathfrak{n}$ denote the nilradical of $R$. Note that $\operatorname{Spec} R$ is homeomorphic to $\operatorname{Spec} R / \mathfrak{n}$. Moreover, $\mathfrak{n}$ supports a Frobenius action and is $F$-torsion. The long exact sequence of local cohomology relating $H_{\mathfrak{m}}^{1}(R)$ and $H_{\mathfrak{m}}^{1}(R / \mathfrak{n})$ implies that if $H_{\mathfrak{m}}^{1}(R / \mathfrak{n})$ is $F$-torsion, then so is $H_{\mathfrak{m}}^{1}(R)$, and hence there is no loss of generality in assuming that $R$ is reduced. Let $R=A /\left(\mathfrak{P}_{1} \cap \cdots \cap \mathfrak{P}_{n}\right)$ where $\mathfrak{P}_{1}, \ldots, \mathfrak{P}_{n}$ are incomparable prime ideals of a power series ring $A=K\left[\left[x_{1}, \ldots, x_{m}\right]\right]$ over a separably closed field $K$. We use induction on $n$ to prove that $H_{\mathfrak{m}}^{1}(R)$ is $F$-torsion; the case $n=1$ follows from Theorem 3.1, so we assume $n>1$ below.

If $\operatorname{dim} R / \mathfrak{p}_{i}=1$ for some $i$, then Spec $R \backslash\{\mathfrak{m}\}$ is the disjoint union of $V\left(\mathfrak{p}_{i}\right) \backslash\{\mathfrak{m}\}$ and $V\left(\mathfrak{p}_{1} \cap \cdots \cap \hat{\mathfrak{p}}_{i} \cap \cdots \cap \mathfrak{p}_{n}\right) \backslash\{\mathfrak{m}\}$, contradicting the connectedness assumption. Hence $\operatorname{dim} R / \mathfrak{p}_{i} \geq 2$ for all $i$. By Remark 2.3, after relabeling the minimal primes if necessary, we may assume that the punctured spectrum of $A / \mathfrak{Q}$ is connected where $\mathfrak{Q}=\mathfrak{P}_{2} \cap \cdots \cap \mathfrak{P}_{n}$. The short exact sequence

$$
0 \rightarrow A /\left(\mathfrak{P}_{1} \cap \mathfrak{Q}\right) \rightarrow A / \mathfrak{P}_{1} \oplus A / \mathfrak{Q} \rightarrow A /\left(\mathfrak{P}_{1}+\mathfrak{Q}\right) \rightarrow 0
$$

induces a long exact sequence of local cohomology modules containing the piece

$$
H_{\mathfrak{M}}^{0}\left(A /\left(\mathfrak{P}_{1}+\mathfrak{Q}\right)\right) \rightarrow H_{\mathfrak{M}}^{1}\left(A /\left(\mathfrak{P}_{1} \cap \mathfrak{Q}\right)\right) \rightarrow H_{\mathfrak{M}}^{1}\left(A / \mathfrak{P}_{1}\right) \oplus H_{\mathfrak{M}}^{1}(A / \mathfrak{Q}) .
$$

Since $\operatorname{rad}\left(\mathfrak{P}_{1}+\mathfrak{P}_{i}\right) \neq \mathfrak{M}$ for some $i>1$, it follows that $\operatorname{dim} A /\left(\mathfrak{P}_{1}+\mathfrak{Q}\right) \geq 1$. Proposition 6.2 now implies that $H_{\mathfrak{M}}^{0}\left(A /\left(\mathfrak{P}_{1}+\mathfrak{Q}\right)\right)$ is $F$-torsion. By the inductive hypothesis, $H_{\mathfrak{M}}^{1}\left(A / \mathfrak{P}_{1}\right)$ and $H_{\mathfrak{M}}^{1}(A / \mathfrak{Q})$ are $F$-torsion as well. The exact sequence (3.2.1) implies that $H_{\mathfrak{M}}^{1}\left(A /\left(\mathfrak{P}_{1} \cap \mathfrak{Q}\right)\right)=H_{\mathfrak{m}}^{1}(R)$ is $F$-torsion.

The following lemma will be used in the proof of Theorem 1.2.

Lemma 3.3. Let $(R, \mathfrak{m})$ be a complete local domain with an algebraically closed coefficient field of positive characteristic. Then $H_{\mathfrak{m}}^{1}(R)_{\mathrm{st}}$, the $F$-stable part of the module $H_{\mathfrak{m}}^{1}(R)$, is zero.

Proof. If $\operatorname{dim} R=0$, then $H_{\mathfrak{m}}^{1}(R)=0$, and if $\operatorname{dim} R \geq 2$, then the assertion follows from Theorem 3.1. The remaining case is $\operatorname{dim} R=1$. Theorem 6.3 implies that $H_{\mathfrak{m}}^{1}(R)_{\text {st }}$ has a vector space basis $\eta_{1}, \ldots, \eta_{r}$ such that $F\left(\eta_{i}\right)=\eta_{i}$.

Let $\eta \in H_{\mathfrak{m}}^{1}(R)_{\text {st }}$ be an element with $F(\eta)=\eta$. Considering $H_{\mathfrak{m}}^{1}(R)$ as the cohomology of a suitable Čech complex, let $\eta$ be the class of $y / x$ in $R_{x} / R=H_{\mathfrak{m}}^{1}(R)$, where $y \in R$ and $x \in \mathfrak{m}$. Since $F(\eta)=\eta$, there exists $r \in R$ such that

$$
\left(\frac{y}{x}\right)^{p}-\frac{y}{x}-r=0
$$

and so $y / x \in R_{x}$ is a root of the polynomial $f(T)=T^{p}-T-r \in R[T]$. The polynomial $\overline{f(T)} \in K[T]$ is separable and $R$ is strictly Henselian, so $f(T)$ splits in $R[T]$. Since $y / x$ is a root of $f(T)$ in the fraction field of $R$, it must then be an element of $R$, and hence $\eta=0$.

Proof of Theorem 1.2. We may assume $R$ to be reduced by Proposition 6.5. First consider the case where the punctured spectrum of $R$ is connected. If $\operatorname{dim} R \geq 2$, then $H_{\mathfrak{m}}^{1}(R)$ is $F$-torsion by Theorem 3.2 , so $H_{\mathfrak{m}}^{1}(R)_{\mathrm{st}}=0$. If $\operatorname{dim} R=1$, then $R$ is a domain, and Lemma 3.3 implies that $H_{\mathfrak{m}}^{1}(R)_{\mathrm{st}}=0$.

We continue by induction on the number of connected components of the punctured spectrum of $R$. If the punctured spectrum of $R$ is disconnected, then $R=$ 
$A /(\mathfrak{A} \cap \mathfrak{B})$ where $(A, \mathfrak{M})$ is a power series ring over the field $K$, and $\mathfrak{A}$ and $\mathfrak{B}$ are radical ideals of $A$ which are not $\mathfrak{M}$-primary, but $\mathfrak{A}+\mathfrak{B}$ is $\mathfrak{M}$-primary. There is a short exact sequence

$$
0 \rightarrow A /(\mathfrak{A} \cap \mathfrak{B}) \rightarrow A / \mathfrak{A} \oplus A / \mathfrak{B} \rightarrow A /(\mathfrak{A}+\mathfrak{B}) \rightarrow 0 .
$$

Since $H_{\mathfrak{M}}^{0}(A / \mathfrak{A})=H_{\mathfrak{M}}^{0}(A / \mathfrak{B})=H_{\mathfrak{M}}^{1}(A /(\mathfrak{A}+\mathfrak{B}))=0$, the resulting exact sequence of local cohomology gives us

$$
0 \rightarrow H_{\mathfrak{M}}^{0}(A /(\mathfrak{A}+\mathfrak{B})) \rightarrow H_{\mathfrak{M}}^{1}(A /(\mathfrak{A} \cap \mathfrak{B})) \rightarrow H_{\mathfrak{M}}^{1}(A / \mathfrak{A}) \oplus H_{\mathfrak{M}}^{1}(A / \mathfrak{B}) \rightarrow 0 .
$$

By Theorem 6.4 , we have a $K$-vector space isomorphism

$$
H_{\mathfrak{m}}^{1}(R)_{\mathrm{st}}=H_{\mathfrak{M}}^{1}(A /(\mathfrak{A} \cap \mathfrak{B}))_{\mathrm{st}} \cong H_{\mathfrak{M}}^{0}(A /(\mathfrak{A}+\mathfrak{B}))_{\mathrm{st}} \oplus H_{\mathfrak{M}}^{1}(A / \mathfrak{A})_{\mathrm{st}} \oplus H_{\mathfrak{M}}^{1}(A / \mathfrak{B})_{\mathrm{st}} .
$$

Since $H_{\mathfrak{M}}^{0}(A /(\mathfrak{A}+\mathfrak{B}))_{\text {st }}=K$ by Proposition 6.2 , the inductive hypothesis completes the proof.

We next record the graded versions of the results proved in this section:

Theorem 3.4. Let $R$ be an $\mathbb{N}$-graded ring of positive dimension, which is finitely generated over a field $R_{0}=K$ of characteristic $p>0$.

(1) If $R$ is a domain with $\operatorname{dim} R \geq 2$, and $K$ is separably closed, then $H_{\mathfrak{m}}^{1}(R)$ is F-torsion.

(2) The module $H_{\mathfrak{m}}^{1}(R)$ is F-torsion if and only if $\operatorname{dim} R \geq 2$ and $\operatorname{Proj} R$ is geometrically connected.

(3) Let $K$ be a perfect field, and let $\bar{K}$ denote its algebraic closure. Then the number of connected components of $\operatorname{Proj}\left(R \otimes_{K} \bar{K}\right)$ is

$$
1+\operatorname{dim}_{K} H_{\mathfrak{m}}^{1}(R)_{\mathrm{st}}=1+\operatorname{dim}_{K}\left(\left[H_{\mathfrak{m}}^{1}(R)\right]_{0}\right)_{\mathrm{st}} .
$$

Proof. (1) Note that $H_{\mathfrak{m}}^{1}(R)$ is a $\mathbb{Z}$-graded $R$-module, and that

$$
F:\left[H_{\mathfrak{m}}^{1}(R)\right]_{n} \rightarrow\left[H_{\mathfrak{m}}^{1}(R)\right]_{n p} \quad \text { for all } n \in \mathbb{Z} .
$$

The module $H_{\mathfrak{m}}^{1}(R)$ has finite length, so all elements of $H_{\mathfrak{m}}^{1}(R)$ of positive or negative degree are $F$-torsion; it remains to show that elements $\eta \in\left[H_{\mathfrak{m}}^{1}(R)\right]_{0}$ are $F$-torsion as well. Let $\eta$ be a element of $\left[H_{\mathfrak{m}}^{1}(R)\right]_{0}$ which is not $F$-torsion. As in the proof of Theorem 3.1, after a change of notation we may assume that

$$
F^{e}(\eta)+r_{1} F^{e-1}(\eta)+\cdots+r_{e} \eta=0
$$

where all $r_{i}$ are in $[R]_{0}=K$, and $r_{e}$ is nonzero. Let $\eta=\left[\left(y_{1} / x_{1}, \ldots, y_{d} / x_{d}\right)\right]$ where $H_{\mathfrak{m}}^{1}(R)$ is regarded as the cohomology of a homogeneous Cech complex. Then there exists $r_{e+1} \in K$ such that $y_{i} / x_{i} \in R_{x_{i}}$ is a root of the polynomial

$$
f(T)=T^{p^{e}}+r_{1} T^{p^{e-1}}+\cdots+r_{e} T+r_{e+1} \in K[T] .
$$

But $f(T)$ is a separable polynomial, so it splits in $K[T]$. The element $y_{i} / x_{i}=y_{j} / x_{j}$ is a root of $f(T)$ in the fraction field of $R$, so it must be one of the roots of $f(T)$ in $K$. It follows that $\eta=0$, which completes the proof of (1).

The proof of (2) is now similar to that of Theorem 3.2 and is left to the reader. For (3), note that $F^{e}\left(H_{\mathfrak{m}}^{1}(R)\right)$ is a $K$-vector space since $K$ is perfect, and that

$$
\operatorname{dim}_{K} H_{\mathfrak{m}}^{1}(R)_{\mathrm{st}}=\operatorname{dim}_{\bar{K}} H_{\mathfrak{m}}^{1}\left(R \otimes_{K} \bar{K}\right)_{\mathrm{st}} .
$$

Thus we may assume $K=\bar{K}$, and the proof is similar to that of Theorem 1.2. 
Remark 3.5. Theorem 3.4(3) generalizes, in the case of positive characteristic, the well-known fact that the number of connected components of $X=\operatorname{Proj} R$ is

$$
\operatorname{dim}_{K} H^{0}\left(X, \mathcal{O}_{X}\right)=1+\operatorname{dim}_{K}\left[H_{\mathfrak{m}}^{1}(R)\right]_{0},
$$

where $R$ is an $\mathbb{N}$-graded reduced ring of positive dimension, which is finitely generated over an algebraically closed field $R_{0}=K$. The point is that in this case the Frobenius is bijective on $\left[H_{\mathfrak{m}}^{1}(R)\right]_{0}$. To see this, let

$$
\eta=\left[\left(\frac{y_{1}}{x_{1}}, \ldots, \frac{y_{d}}{x_{d}}\right)\right] \in\left[H_{\mathfrak{m}}^{1}(R)\right]_{0}
$$

be an element with $F(\eta)=0$, where $H_{\mathfrak{m}}^{1}(R)$ is computed as the cohomology of a suitable Cech complex. Then there exists a homogeneous element $r \in R$ with $\left(y_{i} / x_{i}\right)^{p}=r$ in $R_{x_{i}}$ for all $1 \leq i \leq d$. Such an element $r$ must have degree zero, and hence must be an element of $K$. But then $r^{1 / p} \in K$, and, since $R$ is reduced, $y_{i} / x_{i}=r^{1 / p}$ for all $i$. It follows that

$$
\eta=\left[\left(r^{1 / p}, \ldots, r^{1 / p}\right)\right]=0 .
$$

To complete the argument, note that $\left[H_{\mathfrak{m}}^{1}(R)\right]_{0}$ is a finite dimensional $K$-vector space, and that if $\eta_{1}, \ldots, \eta_{n} \in\left[H_{\mathfrak{m}}^{1}(R)\right]_{0}$ are linearly independent, then so are $F\left(\eta_{1}\right), \ldots, F\left(\eta_{n}\right)$. It follows that $F:\left[H_{\mathfrak{m}}^{1}(R)\right]_{0} \rightarrow\left[H_{\mathfrak{m}}^{1}(R)\right]_{0}$ is surjective.

\section{F-PURITY}

A ring homomorphism $\varphi: R \rightarrow S$ is pure if $\varphi \otimes 1: R \otimes_{R} M \rightarrow S \otimes_{R} M$ is injective for every $R$-module $M$. If $R$ is a ring containing a field of characteristic $p>0$, then $R$ is $F$-pure if the Frobenius homomorphism $F: R \rightarrow R$ is pure. The notion was introduced by Hochster and Roberts in the course of their study of rings of invariants in [HR1, HR2].

Examples of $F$-pure rings include regular rings of positive characteristic and their pure subrings. If $\mathfrak{a}$ is generated by square-free monomials in the variables $x_{1}, \ldots, x_{n}$ and $K$ is a field of positive characteristic, then $K\left[x_{1}, \ldots, x_{n}\right] / \mathfrak{a}$ is $F$-pure.

Goto and Watanabe $[\mathrm{GW}]$ classified one-dimensional $F$-pure rings: let $(R, \mathfrak{m})$ be a local ring of positive characteristic such that $R / \mathfrak{m}=K$ is algebraically closed, $F: R \rightarrow R$ is finite, and $\operatorname{dim} R=1$. Then $R$ is $F$-pure if and only if

$$
\hat{R} \cong K\left[\left[x_{1}, \ldots, x_{n}\right]\right] /\left(x_{i} x_{j} \mid i<j\right) .
$$

Two-dimensional $F$-pure rings have attracted a lot of attention: Watanabe [Wat1] proved that $F$-pure normal Gorenstein local rings of dimension two are either rational double points, simple elliptic singularities, or cusp singularities. He also classified two-dimensional normal $\mathbb{N}$-graded rings $R$ over an algebraically closed field $R_{0}$, in terms of $\mathbb{Q}$-divisors on the curve Proj $R$, [Wat2]. In [MS] Mehta and Srinivas obtained a classification of two-dimensional $F$-pure normal singularities in terms of the resolution of the singularity. Hara completed the classification of twodimensional normal $F$-pure singularities in terms of the dual graph of the minimal resolution of the singularity, [HaN].

The results of Section 3 imply that over separably closed fields, $F$-pure domains of dimension two are Cohen-Macaulay. The point is that if $R$ is an $F$-pure ring, then the Frobenius action $F: H_{\mathfrak{m}}^{i}(R) \rightarrow H_{\mathfrak{m}}^{i}(R)$ is an injective map. 
Corollary 4.1. Let $R$ be a local ring with $\operatorname{dim} R \geq 2$, which contains a field of positive characteristic. If $R$ is F-pure and the punctured spectrum of $R$ is formally geometrically connected, then depth $R \geq 2$.

In particular, if $R$ is a complete local F-pure domain of dimension two, with a separably closed coefficient field, then $R$ is Cohen-Macaulay.

Proof. An $F$-pure ring is reduced, so $H_{\mathfrak{m}}^{0}(R)=0$. By Theorem 3.1, $H_{\mathfrak{m}}^{1}(R)$ is $F$-torsion. Since $R$ is $F$-pure, it follows that $H_{\mathfrak{m}}^{1}(R)=0$.

In the graded case, we similarly have:

Corollary 4.2. Let $R$ be an $\mathbb{N}$-graded ring with $\operatorname{dim} R \geq 2$, which is finitely generated over a field $R_{0}$ of positive characteristic. If $R$ is F-pure and $\operatorname{Proj} R$ is geometrically connected, then $\operatorname{depth} R \geq 2$.

The ring $R$ below is a graded $F$-pure domain of dimension two, and depth one. The issue is that Proj $R$ is connected though not geometrically connected.

Example 4.3. Let $K$ be a field of characteristic $p>2$, and $a \in K$ an element such that $\sqrt{a} \notin K$. Let $R=K[x, y, x \sqrt{a}, y \sqrt{a}]$. The domain $R$ has a presentation

$$
R=K[x, y, u, v] /\left(u^{2}-a x^{2}, v^{2}-a y^{2}, u v-a x y, v x-u y\right),
$$

and if $K^{\text {sep }}$ denotes the separable closure of $K$, then

$$
R \otimes_{K} K^{\mathrm{sep}} \cong K^{\operatorname{sep}}[x, y, u, v] /(u-x \sqrt{a}, v-y \sqrt{a})(u+x \sqrt{a}, v+y \sqrt{a}) .
$$

Using a change of variables, $R \otimes_{K} K^{\mathrm{sep}} \cong K^{\mathrm{sep}}\left[x^{\prime}, y^{\prime}, u^{\prime}, v^{\prime}\right] /\left(x^{\prime}, y^{\prime}\right)\left(u^{\prime}, v^{\prime}\right)$. Since $\left(x^{\prime}, y^{\prime}\right)\left(u^{\prime}, v^{\prime}\right)$ is a square-free monomial ideal, $R \otimes_{K} K^{\text {sep }}$ is $F$-pure and it follows that $R$ is $F$-pure. However, $R$ is not Cohen-Macaulay since $x, y$ is a homogeneous system of parameters with a non-trivial relation

$$
(x \sqrt{a}) y=(y \sqrt{a}) x .
$$

Using the Čech complex on $x, y$ to compute $H_{\mathfrak{m}}^{1}(R)$, we see that it is a 1-dimensional $K$-vector space generated by the element

$$
\eta=\left[\left(\frac{x \sqrt{a}}{x}, \frac{y \sqrt{a}}{y}\right)\right] \in H_{\mathfrak{m}}^{1}(R)
$$

corresponding to the relation above. Given $e \in \mathbb{N}$, let $p^{e}=2 k+1$. Then

$$
F^{e}(\eta)=a^{k} \eta
$$

which is a nonzero element of $H_{\mathfrak{m}}^{1}(R)$. Consequently $H_{\mathfrak{m}}^{1}(R)$ is not $F$-torsion, corresponding to the fact that $\operatorname{Proj} R$ is not geometrically connected.

The corollaries obtained in this section imply that over a separably closed field, a graded or complete local $F$-pure domain of dimension two is Cohen-Macaulay. We record an example which shows that this is not true for rings of higher dimension.

Example 4.4. Let $K$ be a field of characteristic $p>0$, and take

$$
A=K\left[x_{1}, \ldots, x_{d}\right] /\left(x_{1}^{d}+\cdots+x_{d}^{d}\right)
$$

where $d \geq 3$. Let $R$ be the Segre product of $A$ and the polynomial $\operatorname{ring} B=K[s, t]$. Then $\operatorname{dim} R=d$, and the Künneth formula for local cohomology implies that

$$
H_{\mathfrak{m}_{R}}^{d-1}(R) \cong\left[H_{\mathfrak{m}_{A}}^{d-1}(A)\right]_{0} \otimes_{K}[B]_{0} \cong K,
$$

so $R$ is not Cohen-Macaulay. If $p \equiv 1 \bmod d$, then $A$ is $F$-pure by [HR2, Proposition 5.21]; hence $A \otimes_{K} B$ and its direct summand $R$ are $F$-pure as well. 


\section{Algorithmic aspects}

Let $R$ be an $\mathbb{N}$-graded ring, which is finitely generated over a finite field $R_{0}=K$. We wish to determine the number of geometrically connected components of the scheme Proj $R$, i.e., the number of connected components of $\operatorname{Proj}\left(R \otimes_{K} \bar{K}\right)$, or, equivalently, of $\operatorname{Proj}\left(R \otimes_{K} K^{\text {sep }}\right)$. While primary decomposition algorithms such as those of [EHV], [GTZ], or [SY], may be used to determine the connected components of Proj $R$, there is computationally no hope of "determining" the connected components over the algebraic closure, $\bar{K}$. However, simply finding their number is much easier: by Theorem 3.4, this is $1+\operatorname{dim}_{K}\left(\left[H_{\mathfrak{m}}^{1}(R)\right]_{0}\right)_{\mathrm{st}}$. Computing this number involves three steps.

(1) Finding a good presentation of $\left[H_{\mathfrak{m}}^{1}(R)\right]_{0}$;

(2) Determining the Frobenius action on $\left[H_{\mathfrak{m}}^{1}(R)\right]_{0}$ in terms of this presentation;

(3) Computing the dimension of the $F$-stable part, $\left(\left[H_{\mathfrak{m}}^{1}(R)\right]_{0}\right)_{\mathrm{st}}$.

If $R=A / \mathfrak{A}$ for a polynomial ring $A$, we first replace $\mathfrak{A}$ by an ideal that has the same radical as $\mathfrak{A}$, but does not have the homogeneous maximal ideal $\mathfrak{M}$ as an associated prime. This can be done by saturating $\mathfrak{A}$ with respect to $\mathfrak{M}$; if desired, one may simply compute the radical of $\mathfrak{A}$, but this is often computationally expensive. Now, since $\mathfrak{M}$ is not associated to $\mathfrak{A}$, one can find a homogeneous system of parameters $x_{1}, \ldots, x_{d}$ for $R$ such that each $x_{i}$ is a nonzerodivisor on $R$.

The length $\ell$ of $\left[H_{\mathfrak{m}}^{1}(R)\right]_{0}$ may be computed by computing the length of its graded dual $\left[\operatorname{Ext}_{A}^{n-1}(R, A(-n))\right]_{0}$, where $\operatorname{dim} A=n$. Of course, if this length is zero, then $X_{\bar{K}}$ is connected. Consider the Koszul cohomology modules

$$
H^{1}\left(x_{1}^{t}, \ldots, x_{d}^{t} ; R\right)=\frac{\left\{\left(a_{1}, \ldots, a_{d}\right) \in R^{d} \mid a_{i} x_{j}^{t}=a_{j} x_{i}^{t} \text { for all } i<j\right\}}{\left\{\left(r x_{1}^{t}, \ldots, r x_{d}^{t}\right) \mid r \in R\right\}} .
$$

These modules have an $\mathbb{N}$-grading, where for homogeneous elements $a_{i} \in R$, we define the degree of $\left[\left(a_{1}, \ldots, a_{d}\right)\right] \in H^{1}\left(x_{1}^{t}, \ldots, x_{d}^{t} ; R\right)$ as

$$
\operatorname{deg}\left[\left(a_{1}, \ldots, a_{d}\right)\right]=\operatorname{deg} a_{i}-\operatorname{deg} x_{i}^{t},
$$

which is independent of $i$. This ensures that for each $t$, the map

$$
\begin{aligned}
H^{1}\left(x_{1}^{t}, \ldots, x_{d}^{t} ; R\right) & \rightarrow H^{1}\left(x_{1}^{t+1}, \ldots, x_{d}^{t+1} ; R\right) \\
{\left[\left(a_{1}, \ldots, a_{d}\right)\right] } & \longmapsto\left[\left(a_{1} x_{1}, \ldots, a_{d} x_{t}\right)\right]
\end{aligned}
$$

preserves degrees. The module $H_{\mathfrak{m}}^{1}(R)$ is the direct limit of these Koszul cohomology modules, and the assumption that the $x_{i}$ are nonzerodivisors ensures that the maps in the direct limit system are injective. The modules $H^{1}\left(x_{1}^{t}, \ldots, x_{d}^{t} ; R\right)$ may be computed for increasing values of $t$, until we arrive at an integer $N$ such that

$$
\ell\left(\left[H^{1}\left(x_{1}^{N}, \ldots, x_{d}^{N} ; R\right)\right]_{0}\right)=\ell .
$$

This gives us a presentation for $\left[H_{\mathfrak{m}}^{1}(R)\right]_{0}=\left[H^{1}\left(x_{1}^{N}, \ldots, x_{d}^{N} ; R\right)\right]_{0}$, in terms of which we now analyze the Frobenius map. Replacing the $x_{i}$ by their powers if needed, assume that $N=1$. Let

$$
\alpha=\left[\left(a_{1}, \ldots, a_{d}\right)\right] \in\left[H^{1}\left(x_{1}, \ldots, x_{d} ; R\right)\right]_{0},
$$

in which case, $F(\alpha)=\left[\left(a_{1}^{p}, \ldots, a_{d}^{p}\right)\right] \in\left[H^{1}\left(x_{1}^{p}, \ldots, x_{d}^{p} ; R\right)\right]_{0}$. Since the map

$$
\left[H^{1}\left(x_{1}, \ldots, x_{d} ; R\right)\right]_{0} \rightarrow\left[H^{1}\left(x_{1}^{p}, \ldots, x_{d}^{p} ; R\right)\right]_{0}
$$


coming from the direct limit system is bijective, it follows that $a_{i}^{p} \in x_{i}^{p-1} R$ for each $1 \leq i \leq d$. Setting $b_{i}=a_{i}^{p} / x_{i}^{p-1}$, we arrive at

$$
F(\alpha)=\left[\left(b_{1}, \ldots, b_{d}\right)\right] \in\left[H^{1}\left(x_{1}, \ldots, x_{d} ; R\right)\right]_{0} .
$$

Using this description of Frobenius action on the finite dimensional $K$-vector space $\left[H_{\mathfrak{m}}^{1}(R)\right]_{0}=\left[H^{1}\left(x_{1}, \ldots, x_{d} ; R\right)\right]_{0}$, it is now straightforward to compute the ranks of the vector spaces

$$
\left[H_{\mathfrak{m}}^{1}(R)\right]_{0} \supseteq F\left(\left[H_{\mathfrak{m}}^{1}(R)\right]_{0}\right) \supseteq F^{2}\left(\left[H_{\mathfrak{m}}^{1}(R)\right]_{0}\right) \supseteq \ldots,
$$

and hence of the $F$-stable part, $\left(\left[H_{\mathfrak{m}}^{1}(R)\right]_{0}\right)_{\text {st }}$.

\section{Appendix: $F$-torsion modules And $F$-STABle Vector SPACES}

Let $R$ be a commutative ring containing a field $K$ of characteristic $p>0$. A Frobenius action on an $R$-module $M$ is an additive map $F: M \rightarrow M$ such that $F(r m)=r^{p} F(m)$ for all $r \in R$ and $m \in M$. In this case, ker $F$ is a submodule of $M$, and we have an ascending sequence of submodules of $M$,

$$
\text { ker } F \subseteq \operatorname{ker} F^{2} \subseteq \operatorname{ker} F^{3} \subseteq \ldots
$$

The union of these is the $F$-nilpotent submodule of $M$, denoted $M_{\text {nil }}=\bigcup_{e \in \mathbb{N}} \operatorname{ker} F^{e}$. We say $M$ is $F$-torsion if $M_{\text {nil }}=M$.

Proposition 6.1. Let $(R, \mathfrak{m})$ be a local ring containing a field of positive characteristic, and let $M$ be an Artinian $R$-module with a Frobenius action. Then there exists e $\in \mathbb{N}$ such that $F^{e}\left(M_{\text {nil }}\right)=0$.

Hence an Artinian module $M$ is F-torsion if and only if $F^{e}(M)=0$ for some $e$.

Proof. This is proved in [HS, Proposition 1.11] under the hypothesis that $R$ is a complete local ring with a perfect coefficient field. The general case may be concluded from this, but a more elegant approach is via Lyubeznik's theory of F-modules; see [Ly1, Proposition 4.4].

If $R$ is a ring containing a perfect field $K$ of positive characteristic and $M$ is an $R$-module with a Frobenius action, then $F(M)$ is a $K$-vector space, and we have a descending sequence of $K$-vector spaces

$$
F(M) \supseteq F^{2}(M) \supseteq F^{3}(M) \supseteq \ldots .
$$

The $F$-stable part of $M$ is the vector space $M_{\mathrm{st}}=\bigcap_{e \in \mathbb{N}} F^{e}(M)$.

Proposition 6.2. Let $(R, \mathfrak{m}, K)$ be a local ring of dimension $d$ which contains a field of positive characteristic.

(1) $H_{\mathfrak{m}}^{0}(R)$ is F-torsion if and only if $d>0$.

(2) $H_{\mathfrak{m}}^{d}(R)$ is not F-torsion.

(3) If $d=0$ and $K$ is perfect, then $H_{\mathfrak{m}}^{0}(R)_{\mathrm{st}}=R_{\mathrm{st}}=K$.

Proof. (1) If $d=0$, then $H_{\mathfrak{m}}^{0}(R)=R$, which is not $F$-torsion. If $d>0$, then $H_{\mathfrak{m}}^{0}(R)$ is contained in $\mathfrak{m}$. Since every element of $H_{\mathfrak{m}}^{0}(R)$ is killed by a power of $\mathfrak{m}$, it follows that each element is nilpotent. (See also [Ly2, Corollary 4.6(a)].)

(2) View $H_{\mathfrak{m}}^{d}(R)$ as the cohomology of a Cech complex on a system of parameters $x_{1}, \ldots, x_{d}$ for $R$, and let $\eta=\left[1+\left(x_{1}, \ldots, x_{d}\right)\right] \in H_{\mathfrak{m}}^{d}(R)$. For all $e_{0} \in \mathbb{N}$, the collection of elements $F^{e}(\eta)$ with $e>e_{0}$ generates $H_{\mathfrak{m}}^{d}(R)$ as an $R$-module. Hence $F^{e_{0}}(\eta)$ cannot be zero by Grothendieck's nonvanishing theorem. 
(3) Since $\mathfrak{m}$ is nilpotent in this case, for integers $e \gg 0$ we have

$$
F^{e}\left(H_{\mathfrak{m}}^{0}(R)\right)=F^{e}(R)=\left\{x^{p^{e}} \mid x \in R\right\}=\left\{(y+z)^{p^{e}} \mid y \in K, z \in \mathfrak{m}\right\}=K .
$$

Theorem 6.3. Let $(R, \mathfrak{m})$ be a local ring with a perfect coefficient field $K$ of positive characteristic. Let $M$ be an Artinian $R$-module with a Frobenius action. Then $M_{\mathrm{st}}$ is a finite dimensional $K$-vector space, and $F: M_{\mathrm{st}} \rightarrow M_{\mathrm{st}}$ is an automorphism of the Abelian group $M_{\mathrm{st}}$.

If $K$ is algebraically closed, then there exists a $K$-basis $e_{1}, \ldots, e_{n}$ for $M_{\mathrm{st}}$ such that $F\left(e_{i}\right)=e_{i}$ for all $1 \leq i \leq n$.

Proof. For the finiteness assertion, see [HS, Theorem 1.12] or [Ly1, Proposition 4.9]. It is easily seen that $F: M_{\mathrm{st}} \rightarrow M_{\mathrm{st}}$ is an automorphism whenever $M_{\mathrm{st}}$ is finite dimensional. The existence of the special basis when $K$ is algebraically closed follows from [Di, Proposition 5, page 233].

Theorem 6.4 ([HS, Theorem 1.13]). Let $(R, \mathfrak{m})$ be a complete local ring with an algebraically closed coefficient field of positive characteristic. Let $L, M, N$ be $R$ modules with Frobenius actions such that we have a commutative diagram

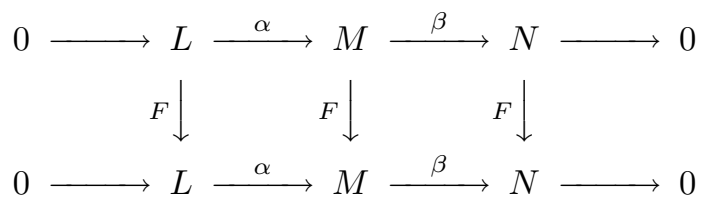

with exact rows. If $L$ is Noetherian and $N$ is Artinian, then the F-stable parts form a short exact sequence

$$
0 \rightarrow L_{\mathrm{st}} \rightarrow M_{\mathrm{st}} \rightarrow N_{\mathrm{st}} \rightarrow 0 .
$$

Proposition 6.5. Let $(R, \mathfrak{m}, K)$ be a complete local ring with an algebraically closed coefficient field of positive characteristic. Let $\mathfrak{n}$ denote the nilradical of $R$. Then for all $i \geq 0$, the natural map $H_{\mathfrak{m}}^{i}(R) \rightarrow H_{\mathfrak{m}}^{i}(R / \mathfrak{n})$, when restricted to F-stable subspaces, gives an isomorphism

$$
H_{\mathfrak{m}}^{i}(R)_{\mathrm{st}} \cong H_{\mathfrak{m}}^{i}(R / \mathfrak{n})_{\mathrm{st}} .
$$

Proof. Let $k$ be an integer such that $\mathfrak{n}^{p^{k}}=0$. The short exact sequence

$$
0 \rightarrow \mathfrak{n} \rightarrow R \rightarrow R / \mathfrak{n} \rightarrow 0
$$

induces a long exact sequence of local cohomology modules

$$
\cdots \rightarrow H_{\mathfrak{m}}^{i}(\mathfrak{n}) \stackrel{\alpha}{\rightarrow} H_{\mathfrak{m}}^{i}(R) \stackrel{\beta}{\rightarrow} H_{\mathfrak{m}}^{i}(R / \mathfrak{n}) \stackrel{\gamma}{\rightarrow} H_{\mathfrak{m}}^{i+1}(\mathfrak{n}) \rightarrow \cdots .
$$

Consider an element $\mu \in \operatorname{ker}(\beta) \cap H_{\mathfrak{m}}^{i}(R)_{\mathrm{st}}$. Then $\mu \in \operatorname{image}(\alpha)$, so $F^{k}(\mu)=0$. The Frobenius action on $H_{\mathfrak{m}}^{i}(R)_{\mathrm{st}}$ is an automorphism, so $\mu=0$, and hence the map $H_{\mathfrak{m}}^{i}(R)_{\mathrm{st}} \rightarrow H_{\mathfrak{m}}^{i}(R / \mathfrak{n})_{\text {st }}$ is injective.

To complete the proof it suffices, by Theorem 6.3 , to consider an element $\eta \in$ $H_{\mathfrak{m}}^{i}(R / \mathfrak{n})_{\text {st }}$ with $F(\eta)=\eta$, and prove that it lies in the image of $H_{\mathfrak{m}}^{i}(R)_{\mathrm{st}}$. Now $\gamma(\eta) \in H_{\mathfrak{m}}^{i+1}(\mathfrak{n})$ so $F^{k}(\gamma(\eta))=0$, and therefore $F^{k}(\eta)=\eta \in \operatorname{ker}(\gamma)$.

Let $\eta=\beta(\mu)$ for some element $\mu \in H_{\mathfrak{m}}^{i}(R)$. Then $\beta(F(\mu)-\mu)=0$, which implies that $F(\mu)-\mu \in$ image $(\alpha)$. Consequently $F^{k}(F(\mu)-\mu)=0$, which shows that $F^{k+1}(\mu)=F^{k}(\mu)$, and hence that $F^{k}(\mu) \in H_{\mathfrak{m}}^{i}(R)_{\text {st }}$. Since

$$
\beta\left(F^{k}(\mu)\right)=F^{k}(\beta(\mu))=F^{k}(\eta)=\eta,
$$

we are done. 


\section{REFERENCES}

[BR] M. Brodmann and J. Rung, Local cohomology and the connectedness dimension in algebraic varieties, Comment. Math. Helv. 61 (1986), 481-490. MR860135 (87j:14006)

[Di] J. Dieudonné, Lie groups and Lie hyperalgebras over a field of characteristic $p>0$. II, Amer. J. Math. 77 (1955), 218-244. MR0067872 (16:789f)

[EHV] D. Eisenbud, C. Huneke, and W. Vasconcelos, Direct methods for primary decomposition, Invent. Math. 110 (1992), 207-235. MR1185582 (93j:13032)

[Fa] G. Faltings, Über lokale Kohomologiegruppen hoher Ordnung, J. Reine Angew. Math. 313 (1980), 43-51. MR552461 (82f:14006)

[GTZ] P. Gianni, B. Trager, and G. Zacharias, Gröbner bases and primary decomposition of polynomial ideals, J. Symbolic Comput. 6 (1988), 149-167. MR988410 (90f:68091)

[GW] S. Goto and K.-i. Watanabe, The structure of one-dimensional F-pure rings, J. Algebra 49 (1977), 415-421. MR0453729 (56:11989)

[HaN] N. Hara, Classification of two-dimensional F-regular and F-pure singularities Adv. Math. 133 (1998), 33-53. MR1492785 (99a:14048)

[HaR] R. Hartshorne, Cohomological dimension of algebraic varieties, Ann. of Math. (2) 88 (1968), 403-450. MR0232780 (38:1103)

[HS] R. Hartshorne and R. Speiser, Local cohomological dimension in characteristic p, Ann. of Math. (2) 105 (1977), 45-79. MR0441962 (56:353)

[HH] M. Hochster and C. Huneke, Indecomposable canonical modules and connectedness, in: Commutative algebra: Syzygies, multiplicities, and birational algebra (South Hadley, MA, 1992), Contemp. Math. 159, Amer. Math. Soc., Providence, RI, 1994, 197-208. MR1266184 (95e:13014)

[HR1] M. Hochster and J. Roberts, Rings of invariants of reductive groups acting on regular rings are Cohen-Macaulay, Advances in Math. 13 (1974), 115-175. MR0347810 (50:311)

[HR2] M. Hochster and J. Roberts, The purity of the Frobenius and local cohomology, Advances in Math. 21 (1976), 117-172. MR0417172 (54:5230)

[HL] C. Huneke and G. Lyubeznik, On the vanishing of local cohomology modules, Invent. Math. 102 (1990), 73-93. MR1069240 (91i:13020)

[Ly1] G. Lyubeznik, F-modules: Applications to local cohomology and D-modules in characteristic $p>0$, J. Reine Angew. Math. 491 (1997), 65-130. MR1476089 (99c:13005)

[Ly2] G. Lyubeznik, On the vanishing of local cohomology in characteristic $p>0$, Compos. Math. 142 (2006), 207-221. MR2197409 (2007b:13029)

[MS] V. B. Mehta and V. Srinivas, Normal F-pure surface singularities, J. Algebra 143 (1991), 130-143. MR1128650 (92j:14044)

[Og] A. Ogus, Local cohomological dimension of algebraic varieties, Ann. of Math. (2) 98 (1973), 327-365. MR0506248 (58:22059)

[PS] C. Peskine and L. Szpiro, Dimension projective finie et cohomologie locale, Inst. Hautes Études Sci. Publ. Math. 42 (1973), 47-119. MR0374130 (51:10330)

[Ra] M. Raynaud, Anneaux locaux henséliens, Lecture Notes in Mathematics 169, SpringerVerlag, Berlin-New York, 1970. MR0277519 (43:3252)

[SY] T. Shimoyama and K. Yokoyama, Localization and primary decomposition of polynomial ideals, J. Symbolic Comput. 22 (1996), 247-277. MR1427183 (98a:13038)

[Wal] U. Walther, Algorithmic determination of the rational cohomology of complex varieties via differential forms, Contemp. Math. 286 (2001), 185-206. MR1874280 (2003b:14027)

[Wat1] K.-i. Watanabe, Study of F-purity in dimension two, in: Algebraic Geometry and Commutative Algebra in honor of Masayoshi Nagata, Vol. II, Kinokuniya, Tokyo, 1988, 791-800. MR977783 (90b:14005)

[Wat2] K.-i. Watanabe, F-regular and F-pure normal graded rings, J. Pure Appl. Algebra 71 (1991), 341-350. MR1117644 (92g:13003)

Department of Mathematics, University of Utah, 155 South 1400 East, Salt Lake City, UTAн 84112

E-mail address: singh@math.utah.edu

Department of Mathematics, Purdue University, 150 N. University Street, West LAFAYETTE, INDIANA 47907

E-mail address: walther@math.purdue.edu 